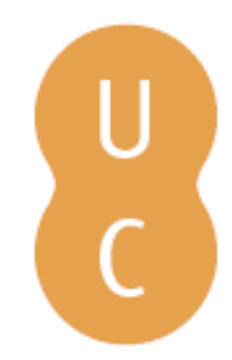

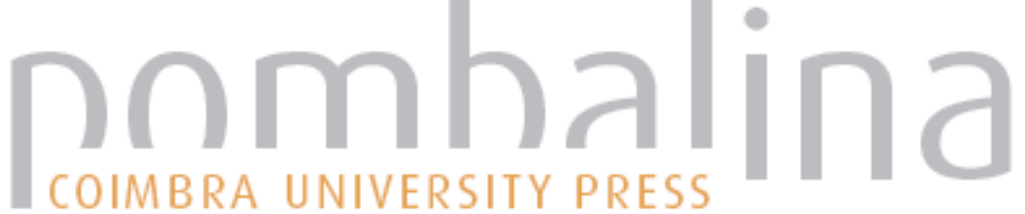

\section{Scripts para leituras infinitas}

Autor(es): Portela, Manuel

Publicado por: Imprensa da Universidade de Coimbra

URL

persistente: URI:http://hdl.handle.net/10316.2/42342

DOI: $\quad$ DOI:https://doi.org./10.14195/978-989-26-1308-6_31

Accessed : $\quad$ 26-Apr-2023 11:03:01

A navegação consulta e descarregamento dos títulos inseridos nas Bibliotecas Digitais UC Digitalis, UC Pombalina e UC Impactum, pressupõem a aceitação plena e sem reservas dos Termos e Condições de Uso destas Bibliotecas Digitais, disponíveis em https://digitalis.uc.pt/pt-pt/termos.

Conforme exposto nos referidos Termos e Condições de Uso, o descarregamento de títulos de acesso restrito requer uma licença válida de autorização devendo o utilizador aceder ao(s) documento(s) a partir de um endereço de IP da instituição detentora da supramencionada licença.

Ao utilizador é apenas permitido o descarregamento para uso pessoal, pelo que o emprego do(s) título(s) descarregado(s) para outro fim, designadamente comercial, carece de autorização do respetivo autor ou editor da obra.

Na medida em que todas as obras da UC Digitalis se encontram protegidas pelo Código do Direito de Autor e Direitos Conexos e demais legislação aplicável, toda a cópia, parcial ou total, deste documento, nos casos em que é legalmente admitida, deverá conter ou fazer-se acompanhar por este aviso.

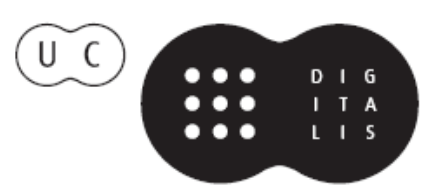





\title{
SCRIPTS PARA LEITURAS INFINITAS
}

\author{
Manuel Portela
}

Resumo: Ao tratar a Internet como a base de dados para textos programados, a série de obras intitulada The Readers Project (2009-2015), de John Cayley e Daniel C. Howe, chama a atenção para a política da linguagem contida na infraestrutura eletrónica e no conjunto dos processos algorítmicos de dadificação dos usos quotidianos da linguagem. Explora as propriedades gerativas da linguagem e a processabilidade em rede do meio digital para interrogar as condições discursivas e materiais de produção de sentido numa cultura medial algorítmica ubíqua. Enquanto exemplo da poética de base de dados, The Readers Project constitui uma poderosa reflexão sobre a linguagem como modo de ação social e sobre a política de processamento linguístico na era da recolha massiva de dados.

${ }^{1}$ Este texto resulta da reescrita de uma comunicação originalmente apresentada no encontro "Chercher le Texte: Locating the Text in Electronic Literature" (organizado pela Electronic Literature Organization, na Universidade de Paris VIII, 24-27 de setembro de 2013). Expresso a minha gratidão aos seus organizadores, em especial a Philippe Bootz. 
Palavras-chave: literatura eletrónica; leitores programados; geradores textuais; poética de base de dados; "détournement".

\begin{abstract}
Treating the Internet as a database for generating new writings, the series of works titled The Readers Project (2009-2015), by John Cayley and Daniel C. Howe, draws attention to the politics of language contained in the electronic infrastructure and in its algorithmic processes for datafying our uses of language. This series explores the generative properties of language and the networked processability of the digital medium to interrogate the discursive and material conditions of meaning production in our pervasive algorithmic media culture. As an example of database poetics, The Readers Project contains a powerful reflection on language as a mode of social action and on the politics of language processing in the age of big data.
\end{abstract}

Keywords: electronic literature; programmed readers; textual generators; database poetics; "détournement".

\title{
1. Escrever (com) a Internet
}

Investigação recente sobre a literatura eletrónica tem tentado descrever a Internet como um espaço de dados para intervenções literárias. Através da análise de obras que fazem uso da rede como meio de produção poética, estes discursos críticos dirigem a atenção para os modos através dos quais a própria infraestrutura da Internet se tornou constitutiva das nossas práticas linguísticas e sociais (ver, por exemplo, Hayles 2012; Funkhouser 2012; Portela 2013; Emerson 2014; Pressman 2014; e Baldwin 2015). Algumas das criações digitais 
analisadas naquelas obras críticas usam e tematizam as novas condições de produção robótica da escrita a dois níveis: em primeiro lugar, adotando elas próprias os algoritmos, protocolos, recursos e interfaces da rede como parte integrante da sua poética material; em segundo lugar, desnaturalizando a transparência comunicacional do meio digital e dirigindo a atenção para o código-fonte, para a retórica específica das ferramentas de software e para as relações económicas incorporadas na tecnologia. ${ }^{2}$

Em Reading Writing Interfaces (2014), Lori Emerson analisa a consciência da interface em vários processos de escrita e em diversos média. A sua abordagem arqueológica à poética medial permite desconstruir a invisibilidade da interface e da ideologia da superfície multitoque "amigável" que se tornou dominante no mundo atual da comunicação mediada por computador e nos paradigmas da engenharia de interação humano-computador. Através da análise literária de uma série de obras, que incluem poesia concreta feita com a máquina de escrever, os fascículos manuscritos de Emily Dickinson e várias obras de literatura eletrónica - de First Screening (1984), de bpNichol, à colheita algorítmica na rede levada a cabo por Cayley e Howe em The Readers Project (2009-2015) -, Emerson mostra a fricção do material da interface como condição necessária para a expressão. A retroação sugerida entre digital e impresso propõe a intermediação como um processo dinâmico e estratificado, contribuindo para uma redefinição crítica tanto do "eletrónico" como da "interface".

De particular importância para as obras programadas que são o tema deste artigo é The Internet Unconscious (2015), de Sandy Baldwin. Baldwin faz uma leitura literária da Internet enquanto

${ }^{2}$ Entre os exemplos mais significativos que, ao longo da última década, tematizam as suas condições socioeletrónicas de produção encontram-se obras de Mary-Anne Breeze (MEZ), John Cayley e Daniel C. Howe, Jim Andrews, Angela Ferraiolo, Eugenio Tisselli e Jody Zeller. 
espaço de escrita e leitura inabarcável e inapreensível, descrevendo a própria Internet como um conjunto distribuído de práticas (do e-mail às redes sociais ao ASCII ao CAPTCHA ao spam) constitutivas quer da net enquanto "literatura eletrónica", quer do sujeito da Internet. Esta tentativa de nomear o inconsciente da literacia eletrónica desloca o foco de atenção de uma explicação estritamente funcional ou estética dos processos de escrita e leitura que ocorrem nos e através dos meios digitais, para uma teorização autorreflexiva sobre Internet, não tanto como um espaço de escrita mas enquanto uma nova forma de escrita, ou seja, enquanto uma série de protocolos de escrita que projetam os nossos atos de escrita e os nossos corpos nesse "além" do ecrã e da sua interface gráfica do utilizador.

A autoprojeção nessa escrita-Internet torna-se assim a introjeção do seu aparelho, ou, por outras palavras, a condição inconsciente da nossa situação telecomunicacional. Colocando retoricamente em primeiro plano o eu que escreve e jogando com a divisão entre o eu que escreve e o eu que é escrito - o "I" recorrente do seu texto realiza-se performativamente como materialização autoconsciente do sujeito escrevente da escrita-Internet -, The Internet Unconscious é também uma instanciação autoexemplificativa do poder do discurso: "Let me state: I write away, as in wear away, one drifting piece of our inconceivable inhabitation of the net. Writing away through myself and toward myself and in lieu of myself. Writing as weapon and spew and nothing." (107)

É enquanto mecanismos para observar este "escrever com" e "ser escrito por" algoritmos e processos automáticos e inconscientes da rede que The Readers Project (Cayley \& Howe, 2009-2015) se torna relevante, na medida em que permite repensar a poética gerativa da base de dados como expressão da condição da linguagem humana na era do processamento automático. A criação literária algorítmica incidiria, por um lado, sobre a crescente dadificação dos usos 
quotidianos da linguagem, que privatiza a palavra e a transforma num dispositivo de vigilância e controlo; e, por outro, sobre a possibilidade de revelar, através da automatização, as estruturas da linguagem enquanto modos de ação. No caso de The Readers Project, a geração de novas instâncias textuais funciona com base em protocolos de leitura formalizados que reassociam elementos de diversos corpora textuais, que vão de um pequeno texto autodelimitado a pesquisas $n$-gram abertas na base infindável de textos da Internet.

\section{Ler (com) a Internet}

Writing only exists as it is read; or as function of its virtual, potential, and intermittent readability; or as function of memory, which is simply a special type of transcription within human readers. (Cayley 2013, n.p.)

The Readers Project (2009-2015) de John Cayley e Daniel C. Howe é o título geral de uma série de obras conceituais e processuais programadas, que exploram a dinâmica da escrita e da leitura em bases de dados em rede. ${ }^{3} \mathrm{O}$ ato de encontrar o texto na base de dados é a sua maneira particular de localizar o literário em ambos os lados da performance literária: como um ato de escrita e como um ato de leitura. Com efeito, o entrelaçamento de escrita e de leitura, que ocorre nesta série de obras, performances e instalações, sugere que o literário pode ser uma forma de leitura que escreve e uma forma de escrita que lê. A reflexividade característica da prática

3 Documentação detalhada sobre as várias iterações do projeto - incluindo performances, instalações e publicações - pode ser encontrada em http://thereadersproject.org/. 
literária poderia então ser descrita como o ciclo de retroalimentação algorítmica entre ler a escrita e escrever a leitura (Cayley 2011; Howe \& Cayley 2011).

O título deste artigo - "scripts para leituras infinitas" - poderia ser reformulado como "scripts para escritas infinitas", na medida em que cada leitura é iterada como novo ato de escrita. É neste campo de forças de transações e interações que nos inventamos como seres humanos: através de performances literárias, agora alargadas ao processamento robótico e ubíquo da linguagem natural. Todavia, aquilo que é mais singular nas intervenções programadas de Cayley e Howe sobre as bases de dados da linguagem não é a produtividade permutacional e poética dos seus algoritmos por si só, mas as suas implicações políticas e cognitivas, uma das quais é a consciência da natureza algorítmica das práticas de escrita e leitura nos meios programáveis em rede. Nas últimas obras, a sua característica reflexividade algorítmica leitura-escrita e escrita-leitura foi programaticamente desenvolvida como intervenção estética e crítica sobre a própria Internet enquanto infraestrutura de escrita-leitura (Howe \& Cayley 2013; Cayley 2013).

A digitalização massiva da escrita que está em curso ocorre dentro de uma infraestrutura tecnológica e económica que tem sido descrita por Cayley e Howe como um processo de "emparcelamento" e "vetorialização" da linguagem natural e da prática simbólica (Howe \& Cayley 2013: 2). Um dos seus objetivos principais, ao fazerem prospeção e recolha nessa gigantesca base de dados da língua inglesa com a ajuda involuntária dos algoritmos de pesquisa da Google, é reivindicar os bens comuns da linguagem escrita contra uma lógica proprietária do discurso. A função das letras minúsculas dos termos de serviço das grandes empresas de dados ["big data"] e de computação em nuvem ["cloud computing"] é naturalizar uma distribuição injusta de recursos no domínio da escrita e das práticas de leitura (Cayley 2013). 
Em How It Is in Common Tongues (2012) - uma das suas intervenções mais significativas sobre a Internet como infraestrutura linguística -, a lógica do copyright no nosso sistema jurídico é posta em causa não apenas enquanto legado da cultura impressa e das anteriores tecnologias dos média, mas também enquanto um já previsível futuro vigiado e emparcelado pelo copyright, que está em vias de ser reconstituído e reforçado na cultura eletrónica. Nesta obra, o texto de How It Is, de Samuel Beckett, é reproduzido através de uma colagem de citações oriundas de fontes eletrónicas não atribuíveis a Beckett, sugerindo que a apropriação autoral e institucional pode sempre reverter para o fluxo comum da linguagem. Este processo de colagem, por sua vez, é obtido por através do détournement e da refuncionalização dos "algorithmic, compositional, and configurative agents of big software's network services" (Howe \& Cayley 2013: 1). Neste caso, Cayley e Howe usam um agente programado de leitura que se move de acordo com o algoritmo da "Expressão Comum Mais Longa" ["Longest-Common Phrase"]. ${ }^{4}$

A estratégia do leitor programado é tratar a base de dados como um arquivo da língua em que é possível recuperar ocorrências de

\footnotetext{
${ }^{4}$ Veja-se a descrição dos autores: "A Longest-Common Phrase (or LCP) is the longest sequence of words, beginning from a specific point in a text, that can be found on the web, not written by the author or about the text in question. The LCP algorithm is the procedure, generally employed by Phrase-Finding Readers, to locate such phrases via queries to public search engines like Google and Bing. The algorithm begins by doing a search for the first $\mathrm{K}$ words of a text, as an exact match (i.e. as a double-quoted string), with the addition of the author's name and title words of the text excluded. If the search returns no results, one word is trimmed from the end of the string and the search is retried (if a $\mathrm{K}$ value of 10 was initially selected, then we search next for an exact match on the first $\mathrm{K}$ minus 1 , or 9 , words). If once again there are no results, the phrase is shortened again by one (to 8 in our example), and the search is repeated. When finally there is a match, the number of results and the list of matching URLs are stored. If for example, the search returns one or more matches for the first 6 words of the text, the next iteration of the algorithm will begin on the seventh word and proceed similarly. The algorithm terminates when the end of the text is reached and all words have thus been included in a matching phrase." (2013, 2-3)
} 
frases e colocações de palavras que correspondem a uma fonte protegida por copyright. A função do motor de busca sequestrado é mediar entre o algoritmo literário da obra e a base de dados geral da língua escrita em inglês. Esta colaboração involuntária mas estruturada, em grandes quantidades de dados, entre algoritmos de busca e um algoritmo de leitura torna-se o princípio de composição da obra de Cayley e Howe, que procura o texto dentro da base de dados da produção verbal em inglês disponível na rede. O processo de prospeção de sequências de palavras idênticas às da matriz culmina na produção de um livro impresso e encadernado em que o texto de Beckett deixa de ser o texto de Beckett, já que a sua voz autoral foi devolvida ao fluxo estocástico e probabilístico da linguagem, agregado roboticamente, a partir de inúmeros servidores da Internet. A definição do copyright e do direito de autor, nos séculos XVIII e XIX, resultou na reificação histórica da originalidade como um marcador jurídico para a atribuição de propriedade numa determinada organização da linguagem impressa. O capitalismo cultural desenvolveu-se com base neste reconhecimento da escrita como forma de propriedade e de capital, e no controle e licenciamento da sua reprodução. 
how it was I quote ${ }^{1}$ before $\mathrm{Pim}$ with ${ }^{2} \mathrm{Pim}$ after $\mathrm{Pim}^{3}$ how it is three parts $\mathrm{I}^{4}$ say it as $\mathrm{I}$ hear it $^{5}$

voice once without ${ }^{6}$ quaqua on all sides ${ }^{7}$ then in me when ${ }^{8}$ the panting stops ${ }^{9}$ tell me again finish telling $\mathrm{me}^{\mathrm{a}}$ invocation

past $^{\mathrm{b}}$ moments old dreams ${ }^{\mathrm{c}}$ back again or fresh like those ${ }^{\mathrm{d}}$ that pass or things things always and ${ }^{\mathrm{f}}$ memories $\mathrm{I}$ say them as $\mathrm{I}^{10}$ hear them murmur ${ }^{11}$ them in the mud

in $^{12}$ me that were without ${ }^{13}$ when the panting stops ${ }^{14}$ scraps of an ancient voice in ${ }^{15}$ me not mine

$\mathrm{my}^{16}$ life last state last version ${ }^{17}$ ill-said ill-heard ill-recaptured ${ }^{18}$ ill-murmured in the $\mathrm{e}^{19}$ mud brief ${ }^{1 \mathrm{a}}$ movements of the lower face losses $^{1 \mathrm{~b}}$ everywhere

recorded $^{1 \mathrm{c}}$ none the less it's ${ }^{1 \mathrm{~d}}$ preferable somehow ${ }^{1 \mathrm{e}}$ somewhere

\footnotetext{
${ }^{1}$ www.nytimes.com/books/first/w/wiesel-sea.html (Aug 14, 2012.1)

${ }^{2}$ www.cameracellularphone.org/tag/device (id. 1)

${ }^{3}$ www.kwarmmeud.com/darknesslakorns/?p=1501 (id. 4)

4www.youtube.lu/watch?v=MAsddbn_2LM (id. 2) ${ }^{5}$ cucurbite.wordpress.com/ (id. 2620000)

${ }^{6}$ gorwathawarband.guildlaunch.com/forums/viewtopic.php?t=8563276 (id. 2)

${ }^{7}$ pplsorce.com/people/Jim_Quaqua/ (id. 3) ${ }^{8}$ celebrityzap.com/AJ_Langer.html (id. 5)

'www.parrothouse.com/hlthcare.html (id. 4290)

"kindle.amazon.com/work/sample?asin=B002HHLW4M\&pr=1\&publisher=A3SWXVW6

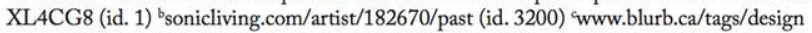

(id. 25) ${ }^{\mathrm{d}}$ www.iraised.it/s/web/index.php?page=7\&q=come-back-quotes (id. 3)

'www.sqlsaturday.com/158/privacypolicy.aspx (id. 7070)

fwww.ilovephilosophy.com/viewtopic.php?f=5\&t=142604\&start=75 (id. 1)

${ }^{10}$ pulsitemeter.com/military/Past-of-Hear.html (id. 2) ${ }^{11}$ www.bartleby.com/84/31.html (id. 29900) ${ }^{12}$ www.facebook.com/dcctowing (id. 7860) ${ }^{13}$ lfpoa.com/headlines/resignation.htm

(id. 68300) ${ }^{14}$ www.cioran63.com/archief76.html (id. 9)

${ }^{15}$ circle.ubc.ca/bitstream/handle/2429/22333/UBC_1980_A1+M37.pdf?sequence=1 (id. 1)

${ }^{16}$ twicsy.com/i/XVig9 (id. 8) ${ }^{17}$ garagepunk.ning.com/profile/deuceloosely (id. 1)

${ }^{18}$ avevilonaso.comyr.com/life-in-forsyth-finnegans-wake.php (id. 1) ${ }^{19} \mathrm{krex} . \mathrm{k}-$

state.edu/dspace/bitstream/2097/9824/1/LD2668R41985B72.pdf (id. 1)

${ }^{12}$ www.highbeam.com/doc/1G1-63262234.html (id. 4430)

${ }^{1 b}$ www.goodreads.com/trivia/submitted/1057191-jason (id. 1)

${ }^{1}$ www.ustream.tv/recorded/24228375 (id. 843) ${ }^{10}$ www.xula.edu/cpsc/cs/megang/ (id. 19600)

${ }^{1 \mathrm{e}}$ Socrates.berkeley.edu/ plab/Palmer_Chap-08.pdf (id. 5710)
}

Figura 1. John Cayley e Daniel C. Howe, How It Is in Common Tongues, p. 7: colher os baldios digitais da linguagem com o algoritmo da Expressão Comum mais Longa. 
Como o texto de Beckett foi montado a partir do fluxo de escritas na Internet, How It Is in Common Tongues (cf. Figura 1) sugere que o texto existe como uma potencialidade nas permutações da língua (tal como instanciado por esta colagem autoexemplificativa colhida na rede), e que são a própria língua e os nossos usos da língua que se tornaram meios de produção da Internet. Em vez de usar a sua demonstração algorítmica das noções de transclusão e transliteratura de Ted Nelson para reforçar o princípio do copyright, How It Is in Common Tongues chama a atenção para os modos como a própria Internet, através das grandes empresas de dados e dos seus termos de serviço, determina as práticas de escrita em rede e emparcela os baldios da linguagem.

Este enquadramento da própria rede como uma construção baseada na língua é uma forma poderosa de sustentar um argumento contra a privatização da linguagem e contra a normalização dos desejos humanos como oportunidades de transação. A língua surge revelada como um capta invisível da rede - o conjunto de dados fornecidos pelas nossas interações de escrita e de leitura diárias -, que passa a fazer parte da lógica publicitária com fins lucrativos e do sistema de vigilância e controlo por palavra-chave. A obra de Cayley e Howe constitui uma intervenção estética sobre as limitações inerentes aos termos de serviço não-recíprocos e aos algoritmos de busca, que eles cooptaram para as suas transações literárias:

As writers and readers we are forced to consider that our relationship with language and literature will never be the same. If the medium of literary art has significantly migrated to the network, where it is gathered, channeled, and filtered by big software on a massive scale, daily touching the linguistic lives of huge populations, then new practices for reading and writing with and against such services must surely arise and go beyond 
any uses that are constrained by the terms of service or use now made unilaterally explicit by contemporary service providers. (Cayley 2013 n.p.)

\section{A língua como infraestrutura}

Se os princípios libertários de How It is in Common Tongues encarnam uma nova prática de leitura e de escrita que aborda a condição algorítmica da linguagem enquanto sequências de dados transacionáveis na era da Internet, The Readers Project tem outras implicações importantes para a produção do literário na rede dos média programáveis e para uma compreensão da performatividade do ato de ler na produção do literário. Algumas das instalações mostram agentes programados a fazer percursos de leitura sobre campos textuais de acordo com determinadas estratégias. Embora não estejam a tentar simular uma prática de leitura particular ou o processamento ocular real do texto à medida que os olhos se movem de um ponto para outro, os vários algoritmos de leitura dão ao espetador uma perceção material da natureza dinâmica da leitura. A leitura atravessa um campo de signos e esta travessia vai criando suas próprias constelações lineares e multilineares. Cada vetor de leitura realiza uma iteração de um comportamento ou estratégia de leitura particular e, deste modo, torna-se uma inscrição na superfície textual. Cada uma destas inscrições é o resultado da execução de scripts que constituem um determinado código de leitura maquínico (Portela 2013: 345-347).

Estes modos de leitura, que se inscrevem num determinado campo textual de acordo com uma estratégia de leitura programada, são experimentações formais com profundas implicações na localização do literário. Nas diversas iterações de 
The Readers Project, o "literário" poderia ser localizado nos seguintes níveis:

1. como a codependência de leitura e escrita: a escrita existe através de uma intervenção que se reinscreve no campo textual de acordo com uma interação corporizada, o que significa que a escrita literária é coproduzida pela leitura literária - a "materialidade performativa" (Drucker 2013) do literário como um modo particular de leitura;

2. como uma instanciação reflexiva do processamento algorítmico da escrita e da leitura em ambientes de rede programados: o algoritmo gerador da obra sustenta uma experiência de leitura-escrita, alimentando-se das bases de dados e dos algoritmos da própria rede - o literário como um modo particular de reescrever a rede e de tornar explícitas as relações contratuais desiguais que regulam o processamento da linguagem cibernética nos atuais sistemas mediais;

3. como uma série de scripts, ou seja, como uma série de regras ou procedimentos que podem ser formalmente codificados num programa para gerar novas instâncias da escrita a partir de permutações linguísticas do arquivo da língua escrita - a literatura como a exploração aberta das combinatórias de estruturas da língua mediadas pela memória arquivada da escrita;

4. como "geradores de texto de diferentes escalas" (Montfort 2012) através dos quais os algoritmos de leitura colhem, destacam, sublinham, deformam, remisturam e citam vários sistemas textuais (desde um determinado texto ou obra até ao corpus inteiro dos textos pesquisáveis em língua inglesa) de acordo com uma regra formal que produz escrita;

5. como uma "ficção cognitiva" (Tabbi 2002), ou seja, como um sistema de auto-organização que representa os seus 
próprios mecanismos; neste caso, o funcionamento dos loops estranhos que ligam mediações linguísticas e mediações programadas na rede eletrónica. A alteridade da Internet como um sistema puramente medial é ressignificada como um sistema linguístico dependente da linguagem natural. A linguagem seria assim um dos elementos infraestruturais constitutivos do inconsciente da rede.

Uma vez que estas leituras da máquina são reoferecidas como escritas a leitores humanos (distintos dos leitores maquínicos que percorrem as sequências de caracteres), a escrita da leitura e a leitura como escrita, contida em The Readers Project, transformam os leitores em metaleitores obrigados a ler o seu próprio ato de ler a leitura do programa. O movimento de leitura, que é retoricamente encenado dentro da superfície textual através da animação textual, é também uma chamada de atenção percetual sobre a cinética contida no ato de mover os olhos de letra em letra e de palavra em palavra. Uma vez que os leitores programados dentro do texto oferecem o seu modo particular de ler como mais um exemplo de escrita para ser lida, ainda uma vez mais, por um leitor humano, as leituras propriamente ditas não podem ser espelhadas ou capturadas exatamente pelos leitores programados, apenas podem ser retomadas como encontros renovados com sinais. O modo particular de leitura programado na máquina de leitura e o ato humano de ler esse modo particular de leitura gera uma posição cognitiva. A escrita gerativa automática é apresentada como um ato de leitura que reescreve o texto e o torna disponível para leitura literária.

Os algoritmos de Cayley e Howe para definir os vários vetores de leitura conseguem evitar a entropia de uma seleção de palavras totalmente aleatória, conseguindo ainda assim espaço para associações inesperadas e improváveis. O efeito poético é produto dessa tensão entre todo o corpus de palavras no texto escolhido como 
base de dados da obra e as regras de vizinhança que governam leituras particulares. Configurado como uma instalação para ser lida por leitores humanos, o processamento estritamente semiótico, baseado em regras de associação de palavras e executado pela máquina, entra nos processos humanos de substituição simbólica e de apreensão afetiva. O movimento textual, que tem lugar no texto que se lê a si mesmo, entra em conflito com o movimento de leitura que analisa e segmenta o texto ao ritmo de um leitor humano. Uma vez que cada leitura maquínica é também uma nova instância da escrita disponível para um leitor humano, o ato de ler a leitura da máquina torna-se um modelo para a iterabilidade infinita da escrita tal como atualizada por cada ato de leitura. A iterabilidade do código é utilizada para sublinhar o ato de ler como um campo de possibilidades fundadas na iterabilidade e na citabilidade da escrita.

As obras de Cayley e Howe estão baseadas numa interação autopoiética com a rede como um sistema de notações de grande escala, onde a própria língua natural - e não apenas a infraestrutura de engenharia das comunicações (o seu hardware e o seu software) - é uma parte essencial dos meios de produção que sustentam a Internet como a rede discursiva e o sistema de notação típico do século XXI. O seu trabalho aponta para este acoplamento quase invisível entre a rede da linguagem natural e a rede de dados codificados. A intervenção de The Readers Project sobre a rede programável é uma intervenção sobre a base de dados da língua em rede. A reflexividade dos seus protocolos de leitura-escrita cria um meta-representação da Internet como uma rede linguística. Esta espécie de equivalente eletrónico da metalepse literária permite um movimento de vai e vem entre a linguagem como significado e a linguagem como conjunto de dados. A iteração maquínica dos comportamentos de leitura é um modo de dar forma literária à nossa base de dados de inscrições digitais, uma tentativa de encontrar o texto no funciona- 
mento automatizado das atuais redes de média e nas manifestações sintomáticas do inconsciente da Internet.
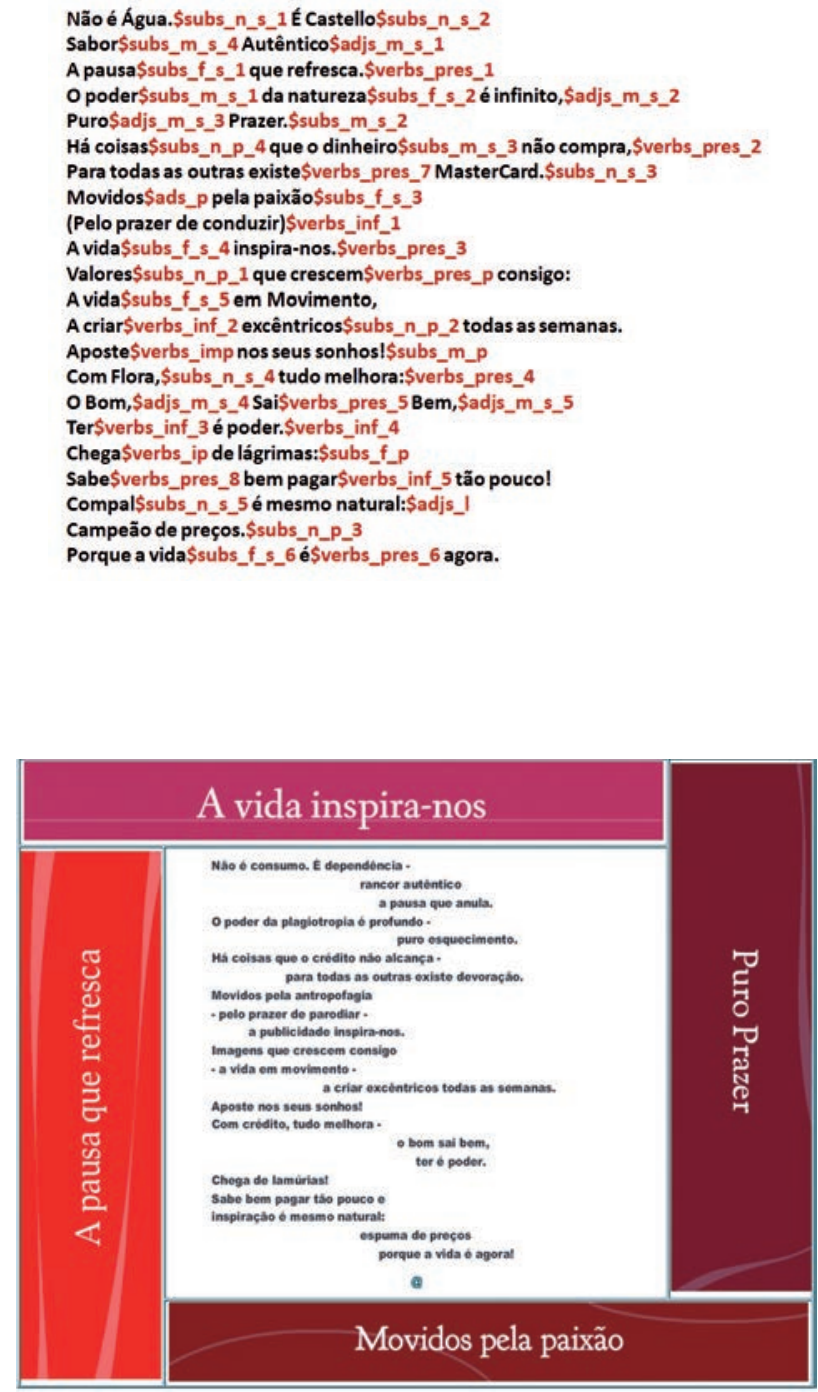


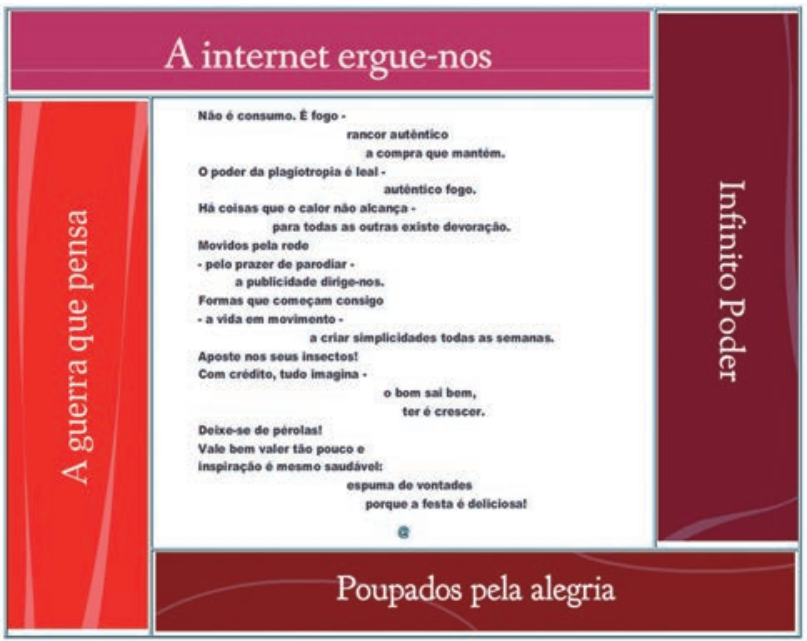

\section{Obras citadas}

Baldwin, S. The Internet Unconscious: On the Subject of Electronic Literature. London: Bloomsbury, 2015. Print.

Cayley, J. "Writing to Be Found and Writing Readers." Digital Humanities Quarterly 5.3 (2012) n.p. Web. 22 Sept. 2015.

—. "Terms of Reference \& Vectorialist Transgressions: Situating Certain Literary Transactions over Networked Services." Amodern 2 (2013) n.p. Web. 22 Sept. 2015.

Cayley, J. \& D.C. Howe. How It Is in Common Tongues. Providence, RI: The Natural Language Liberation Front, 2012. Print.

—. The Readers Project. 2009-2015. Web. 22 Sept. 2015.

Drucker, J. "Performative Materiality and Theoretical Approaches to Interface." Digital Humanities Quarterly 7.1 (2013): n.p. Web. 22 Sept. 2015.

Emerson, L. Reading Writing Interfaces: From the Digital to the Bookbound. Minneapolis, MN: University of Minnesota Press, 2014. Print.

Funkhouser, C.T. New Directions in Digital Poetry. London: Continuum, 2012. Print.

Hayles, N. K. How We Think: Digital Media and Contemporary Technogenesis. Chicago: Chicago University Press, 2012. Print.

Howe, D. C. \& J. Cayley. "The Readers Project: Procedural Agents and Literary Vectors." Leonardo 44.4 (2011): 317-24. Print. 
—. "Reading, Writing, Resisting: Literary Appropriation in the Readers Project." Eds. K. Cleland, L. Fisher \& R. Harley, R. Proceedings of the 19th International Symposium of Electronic Art. ISEA 2013, Sydney. 1-4. Web. 22 Sept. 2015.

Montfort, N. "XS, S, M, L: Creative Text Generators of Different Scales." A Technical Report from the Trope Tank. MIT, January 2012. Trope-12-02. Web. 22 Sept. 2015.

Scripting Reading Motions: The Codex and the Computer as Self-Reflexive Machines. Cambridge, MA: MIT Press, 2013. Print.

Pressman, J. Digital Modernism: Making It New in New Media. Oxford: Oxford University Press, 2014. Print.

Tabbi, J. Cognitive Fictions. Minneapolis, MN: University of Minnesota Press, 2002. Print. 